\title{
Phase I Study of Nab-Paclitaxel plus Gemcitabine as Neoadjuvant Therapy for Borderline Resectable Pancreatic Cancer
}

\author{
KEN-ICHI OKADA, SEIKO HIRONO, MANABU KAWAI, MOTOKI MIYAZAWA, ATSUSHI SHIMIZU, \\ YUJI KITAHATA, MASAKI UENO, SHINYA HAYAMI and HIROKI YAMAUE
}

Second Department of Surgery, Wakayama Medical University, Wakayama, Japan

\begin{abstract}
Background/Aim: The aim of this study was to investigate the safety and feasibility of neoadjuvant nabpaclitaxel plus gemcitabine therapy for patients with borderline resectable pancreatic carcinoma (BRPC). Patients and Methods: The study was a prospective single-center phase I trial for patients with BRPC. The primary endpoint was the toxicity, and secondary endpoints were the resection rate, the $R O$ resection rate and quality of life (QOL) regarding the peripheral sensory neuropathy (PSN). This trial was registered on the UMIN Clinical Trials Registry (UMIN000018382) and on ClinicalTrials.gov (NCT02506803). Results: The overall rate of any grade and grade 3-4 events (CTCAE ver. 4.0 criteria) were $100 \%$ and $90 \%$. The majority of these adverse events represented expected neutropenia. The resection and RO resection rates were $80 \%$ and $70 \%$, respectively. Conclusion: We found that neoadjuvant nab-paclitaxel plus gemcitabine therapy was safe and feasible without stringent selection of patients with BRPC.
\end{abstract}

Pancreatic cancer has extremely poor prognosis among digestive cancers as it spreads rapidly and is rarely detected in its early stage $(1,2)$. A multidisciplinary approach with R0 surgical resection and completion of adjuvant therapy is necessary for favorable survival time $(1,2)$. Therefore, patients with a high risk of R1 status at the surgical margin have been categorized into the borderline resectable pancreatic carcinoma (BRPC) to be selected for a candidacy of neoadjuvant therapy to obtain a better $\mathrm{R} 0$ resection rate $(3,4)$. A recent study by Chaterdee et al. reported that

Correspondence to: Dr. Hiroki Yamaue, Second Department of Surgery, Wakayama Medical University, Wakayama, Japan. Tel: +81 734410613, Fax: +81 73446-6566, e-mail: yamaue-h@wakayamamed.ac.jp

Key Words: Neoadjuvant chemotherapy, borderline resectable, pancreatic cancer. patients who had pathologic complete response or minimal residual tumor showed favorable survival rates compared to patients who had moderate to poor response (5).

These days, for patients with metastatic pancreatic cancer, the introduction of FOLFIRINOX or nab-paclitaxel plus gemcitabine therapies, known for its promising rapid response and safety has attracted many surgeons who in turn adopt these therapies as a neoadjuvant therapy for BRPC (6, 7). FOLFIRINOX therapy should be applied as a neoadjuvant therapy to restrictively selected patients because of the high incidences of severe cholangitis, liver abscess or variants of UGT1A1 which have been reported to intensify myelosuppression such as severe neutropenia (8-10). Recent studies reported the safety and efficacy of modified FOLFIRINOX regimen as a neoadjuvant therapy in selected patients (11-13).

From this point of view, surgeons should pay attention to the balance of benefits and harms for the stronger regimen of neoadjuvant therapy. Hence, there is a demand for new regimens with stronger oncological outcome without restrictions or limitations for safe and effective preoperative therapy (14). In spite of the proven benefits, in the previous report the pancreatic tumors were assessed as non-targeted lesions. Also, the histopathological impact on the tumor cell destruction of BRPC in terms of $\mathrm{R} 0$ resection still remains controversial (15). Notably, the incidence and frequency of severe neutropenia or peripheral sensory neuropathy (PSN) may affect the treatment schedule, dosage of administration, or perioperative quality of life (QOL) of BRPC patients in the limited preoperative period. Therefore, the aim of this study is to investigate the safety and feasibility of nab-paclitaxel plus gemcitabine therapy as a neoadjuvant chemotherapy.

\section{Patients and Methods}

Patients' characteristics. Criteria of eligible patients enrolled in this study were histologically or cytologically confirmed pancreatic adenocarcinoma or adenosquamous carcinoma, an Eastern Cooperative 
Oncology Group (ECOG) PS of 0 or 1, age between 20 and 80 years old, had, a BRPC according to the NCCN clinical practice guideline Version 1. 2014 (16). Pancreatic body carcinoma indicated for distal pancreatectomy with en bloc celiac axis resection (DP-CAR) was determined to be eligible for this trial $(17,18)$. Otherwise, eligibility criteria were the same as described previously (15).

Study design. The study was a prospective single center phase I trial with BRPC. The trial was approved by the institutional review board of Wakayama Medical University (No. 1657). This trial is registered on the UMIN Clinical Trials Registry (UMIN000018382) and on ClinicalTrials.gov (NCT02506803). The patients underwent neoadjuvant chemotherapy as outpatients. The final analysis would be carried out after discharge of the last patient.

Treatment protocol. Enrolled patients were administered a 30-min intravenous infusion of nab-paclitaxel at a dose of $125 \mathrm{mg} / \mathrm{m}^{2}$, followed by a $30-\mathrm{min}$ intravenous infusion of gemcitabine at a dose of $1000 \mathrm{mg} / \mathrm{m}^{2}$, on day one, eight and fifteen over a four-week period as one cycle of regimen (15). If the dose on eighth day was skipped, the next dose was still administered on day fifteen of the same cycle as originally scheduled. All patients were given one week of rest between two cycles. This regimen was repeated twice based on the result of a previous study which reported that the median time to response a 43.0 days (15). Prior to the study treatment, a 5-HT3 receptor antagonist and dexamethasone were given. Selective neurokinin-1 receptor antagonistic antiemetics were recommended to reduce the degree of nausea and vomiting. The treatment was repeated until disease progression or toxicity levels became unacceptable, or when discontinuation was decided by the investigators or by patient refusal. Chemotherapy was restarted when patients' recovery status fulfilled the following criteria: neutrophil count at $>1,500 / \mathrm{mm}^{3}$, platelet count at $>75,000 / \mathrm{mm}^{3}$, total bilirubin at $<1.5 \mathrm{mg} / \mathrm{dl}$, grade $1-2$ peripheral sensory neuropathy and grade 1-2 diarrhea. When the predefined toxic events in the protocol occurred, dose adjustment was required. During the two to eight week rest period, preoperative restaging scans and staging laparoscopy were performed upon completion of therapy. In the absence of disease progression, patients underwent planned pancreatectomy.

Primary and secondary endpoints. The primary endpoint was the toxicity of nab-paclitaxel plus gemcitabine therapy as a neoadjuvant chemotherapy for BRPC patients throughout the entire period of protocol treatment. Secondary endpoints were the resection rate, the R0 resection rate, postoperative morbidity, and QOL regarding the PSN assessed by the questionnaire of FACT/GOG-NTX subscale (Version 4) (Japanese version) (19). Pathologic response was graded according to the system developed by Evans et al. (20) R1-status was defined as the presence of tumor cells and R0-status as the absence of tumor cells at the resection margin.

Adverse events and dose modifications. Toxicities and adverse events were evaluated in accordance with the National Cancer Institute Common Terminology Criteria for Adverse Events [CTCAE] version $4.0(21)$. The criteria for dose reduction and discontinuation of the protocol treatment were followed as previously reported (15). The date of each administration during two cycles was determined as being fixed scheduled regardless of skipping of administration.

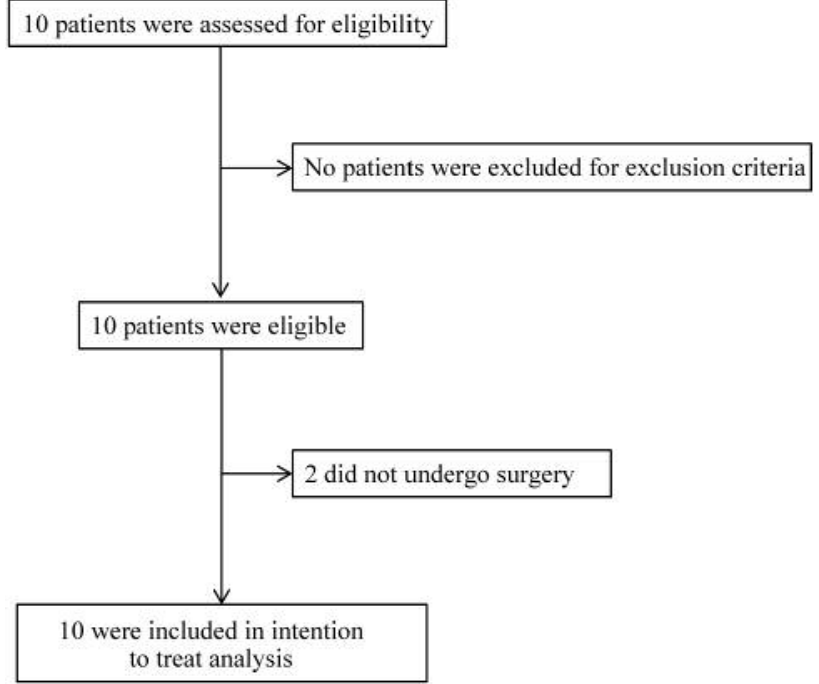

Figure 1. CONSORT flow diagram used in this study.

Assessments. Complete blood counts and differential count of leukocytes, blood chemical tests and physical examinations were carried out at least once a week until the end of the two cycles and every two weeks thereafter. In cases of grade 4 hematological toxicity, re-examination within four days was required. Computed tomography was carried out when the tumor marker was extremely elevated. Tumor response was reviewed in accordance with Response Evaluation Criteria in Solid Tumors (RECIST) version 1.1.

Postoperative complications. The global morbidity rate and type of complications were evaluated by the Dindo's classification (22). Mortality was defined as any deaths related to surgery.

Statistical analyses. The statistical analysis methods used were as previously described (15). Dose intensity of each drug was calculated based on the data from protocol therapy. A value of $p<0.05$ was considered significantly different. All of the analyses were performed using the SPSS II statistical software package (version 20.0; SPSS Inc., Chicago, IL, USA).

\section{Results}

Patient characteristics. Between July 2015 and November 2015, 10 patients were assessed for eligibility. Among them, no patients were excluded based on the eligibility criteria. Tumor response evaluated in accordance with RECIST revealed $\operatorname{SD}(n=9)$ and $\operatorname{PD}(n=1)$. Two patients did not undergo surgery, one due to disease progression with liver metastasis $(n=1)$ and the other due to circumferential invasion of the nervous plexus around the superior mesenteric artery $(n=1)$. All 10 patients received the nab-paclitaxel plus gemcitabine therapy; thus, all 10 patients were included in the safety analysis (Figure 1). The patient characteristics at baseline are shown in Table I. The median age was 70 years 
Table I. Baseline and operative characteristics of patients. Values are mean \pm standard deviation or number, unless otherwise stated.

\begin{tabular}{|c|c|}
\hline Characteristic & $\mathrm{N}$ \\
\hline \multicolumn{2}{|l|}{ Baseline } \\
\hline Gender (male/female) & $6 / 4$ \\
\hline Age, years & $70 \pm 7.4$ \\
\hline Location of pancreatic cancer (Body-Tail/Head) & $2 / 8$ \\
\hline Comorbidity, patient no & 0 \\
\hline Diabetes Mellitus & 4 \\
\hline Hypertension & 5 \\
\hline Biliary stent or drainage & 2 \\
\hline Frequency of administration & $4.6 \pm 0.8$ \\
\hline \multicolumn{2}{|l|}{ Abutment vessel (with overlapping) } \\
\hline Portal vein & 5 \\
\hline Artery (SMA/CA) & $8(6 / 2)$ \\
\hline \multicolumn{2}{|l|}{ UICC-Stage } \\
\hline IB & 1 \\
\hline IIA & 1 \\
\hline IIB & 2 \\
\hline III & 6 \\
\hline Discontinuation of protocol & 2 \\
\hline \multicolumn{2}{|l|}{ Response evaluation } \\
\hline PR & 0 \\
\hline SD & 9 \\
\hline $\mathrm{PD}$ & 1 \\
\hline Decrease rate of CA 19-9 value & $0.49 \pm 1.0$ \\
\hline Resection rate $(\%)$ & 80 \\
\hline \multicolumn{2}{|l|}{ Operative procedure } \\
\hline Pancreaticoduodenectomy & 7 \\
\hline DP-CAR & 1 \\
\hline \multicolumn{2}{|l|}{ Combined resection } \\
\hline Other organ & 1 \\
\hline Portal vein & 4 \\
\hline Artery & 1 \\
\hline Operative time (min) & $407 \pm 79$ \\
\hline Transfusion & 1 \\
\hline Adjuvant chemotherapy with S-1 & 7 \\
\hline Completion & 3 \\
\hline
\end{tabular}

SMA, Superior mesenteric artery; CA, celiac axis; PR, partial response; $\mathrm{SD}$, stable disease; $\mathrm{PD}$, progressive disease; $\mathrm{CA}$, carbohydrate antigen; DP-CAR, distal pancreatectomy with en-bloc celiac axis resection; S1, TS-1; Taiho Pharmaceutical Co., Ltd., Tokyo, Japan).

(range, 57-79). All patients had an ECOG PS 0. The primary site of the tumor was the head of the pancreas in $80 \%$ of the patients, and $20 \%$ of the patients had a biliary stent.

The mean frequency of administration was $4.6 \pm 0.8$ during 2 cycles of regimen. The median dose intensity of nabpaclitaxel and gemcitabine was $83 \%$ and $83 \%$, respectively. There was no delayed duration between the last chemotherapy and operative days in terms of protocol.

Adverse events. Adverse drug reactions deemed to be potentially related to the trial protocol are shown in Table II. The overall rate of any grade events (CTCAE ver. 4.0
Table II. Toxicity following treatment with neoadjuvant nab-paclitaxel plus gemcitabine therapy. Values are number of events. Safety was evaluated in accordance with the Common Terminology Criteria for Adverse Events version 4.0 (21).

\begin{tabular}{lccc}
\hline Treatment toxicity & All grade & G3 & G4 \\
\hline Leukopenia & 9 & 5 & 0 \\
Anemia & 7 & 1 & 0 \\
Thrombocytopenia & 8 & 2 & 0 \\
Neutropenia & 10 & 5 & 4 \\
Liver dysfunction & 5 & 1 & 0 \\
Appetite loss & 3 & 0 & 0 \\
Nausea & 2 & 0 & 0 \\
Vomit & 1 & 0 & 0 \\
Diarrhea & 0 & 0 & 0 \\
Fatigue & 3 & 0 & 0 \\
Oral inflammation & 2 & 1 & 0 \\
Hand foot syndrome & 2 & 1 & 0 \\
Hair loss & 10 & 2 & 0 \\
Febrile neutropenia & 0 & 0 & 0 \\
Cholangitis & 1 & 0 & 0 \\
Interstitial pneumonia & 0 & 0 & 0 \\
Peripheral sensory Neuropathy & 3 & 1 & 0 \\
\hline
\end{tabular}

criteria) during the protocol treatment was $100 \%$. The overall rate of grade 3 and 4 events was $90 \%$. The majority of these adverse events represented expected neutropenia $(n=9)$. All patient data with grade 3-4 neutropenia were recovered to a grade $<3$ by G-CSF within two weeks. However, there were no incidences of serious adverse events (SAE) such as febrile neutropenia, sepsis, grade three or higher interstitial pneumonia, peripheral sensory neuropathy $(n=1)$ or several adverse events of more than grade 3 were observed (Table II). There were no treatment-related deaths in this study.

QOL assessment. The QOL assessment regarding peripheral sensory neuropathy using the questionnaire of FACT/GOGNTX subscale (additional concern) is shown in Table III. All patients except one answered "Not at all" for all questions, but the remaining patient answered "Quite a bit" to "Very much" for 9 of 11 questions. This patient presented grade 3 PSN within the first week of the treatment.

Postoperative complications. Table IV shows the perioperative complications in the groups. Treatment-related SAE occurred in a patient who presented gastric ischemia on postoperative day 5 after DP-CAR. This patient underwent reoperation for total gastrectomy and recovered soon. This case was assessed as a grade IV morbidity of the Dindo's classification.

Resection and $R 0$ resection rates. The resection rate was $80 \%$ in the present study. Two patients did not undergo surgery due to occurrence of distant metastasis and the exacervation of local invasion. Table IV shows the results of 
Table III. FACT/GOG-NTX (v 4.0) additional concerns.

\begin{tabular}{|c|c|c|c|c|c|c|}
\hline \multirow[t]{2}{*}{ Question } & & \multicolumn{5}{|c|}{ Degree } \\
\hline & & 0 & 1 & 2 & 3 & 4 \\
\hline NTX1 & I have numbness or tingling in my hands & 0 & 0 & 0 & 0 & 1 \\
\hline NTX2 & I have numbness or tingling in my feet & 0 & 0 & 0 & 0 & 1 \\
\hline NTX3 & I feel discomfort in my hands & 0 & 0 & 0 & 0 & 1 \\
\hline NTX4 & I feel discomfort in my feet & 0 & 0 & 0 & 1 & 0 \\
\hline NTX5 & I have joint pain or muscle cramps & 0 & 0 & 0 & 0 & 1 \\
\hline HI12 & I feel weak all over & 0 & 0 & 0 & 1 & 0 \\
\hline NTX6 & I have trouble hearing & 0 & 0 & 0 & 0 & 0 \\
\hline NTX7 & I get a ringing or buzzing in my ears & 0 & 0 & 0 & 0 & 0 \\
\hline NTX8 & I have trouble buttoning buttons & 0 & 0 & 0 & 0 & 1 \\
\hline NTX9 & I have trouble feeling the shape of small objects when they are in my hand & 0 & 0 & 0 & 1 & 0 \\
\hline An6 & I have trouble walking & 0 & 0 & 0 & 0 & 1 \\
\hline
\end{tabular}

0 , Not at all; 1 , a little bit; 2 , somewhat; 3 , quite a bit and 4 , Very much.

R0 resection rates of this series and the intraoperative peritoneal cytology. The examination of the histopathological treatment effect based on the Evans grade revealed grade I $(n=3)$, IIa $(n=5)($ Table IV).

\section{Discussion}

The introduction of nab-paclitaxel plus gemcitabine therapy for patients with metastatic pancreatic cancer is attractive to surgeons as this therapy may yield rapid response and improved safety (7). This therapy is therefore used as a neoadjuvant therapy for BRPC patients. We found that neoadjuvant chemotherapy with nab-paclitaxel plus gemcitabine was safe and feasible in terms of adverse events and resection rate (23). The present findings are consistent with those from the Phase I/II study of nab-paclitaxel plus gemcitabine with metastatic pancreatic carcinoma regarding the frequency of neutropenia (15). Despite the high incidence of severe neutropenia, there was no incidence of serious adverse effects such as febrile neutropenia, sepsis, or interstitial pneumonia. Notably, all of the patients who were assessed for eligibility could be enrolled in this study. In contrast to the FOLFIRINOX regimen, there were less concerns about severe cholangitis, liver abscess, and variants of UGT1A1, which have been reported to intensify myelosuppression such as severe neutropenia (24). In the present study, neutropenia was a controllable condition and recovery rate was quick by G-SCF. These facts reflect the better feasibility of applying this regimen to patients with BRPC than the other known regimen in the clinical setting. However, dose modification of nab-paclitaxel plus gemcitabine is not necessarily negative on survival (25) and skipping once or twice should be taken into consideration if we apply original nabpaclitaxel plus gemcitabine regimen to neoadjuvant therapy so not to reduce the efficacy of treatment.
Table IV. Surgical and histopathological results. Values are mean \pm standard deviation or number, unless otherwise stated.

\begin{tabular}{lc}
\hline Surgical outcome & $\mathrm{N}$ \\
\hline Morbidity (Dindo's classification) & \\
Grade I, II & 6 \\
Grade IIIa & 1 \\
Grade IIIb & 0 \\
Grade IV & 1 \\
Grade V & 0 \\
Interval duration between last chemotherapy & \\
and operative day, days & $32 \pm 7.3$ \\
Mortality, no. (\%) & 0 \\
R0 rate (\%) & 70.0 \\
R0 rate in resection case (\%) & 87.5 \\
Intraoperative peritoneal cytology & 9 \\
Positive & 2 \\
Negative & 7 \\
Evans grade & \\
I & 3 \\
IIa & 5 \\
IIb & 0 \\
III & 0 \\
\hline
\end{tabular}

Regarding PSN, although the previous phase I/II study reported the median time to develop grade 3 PSN was 108.5 days, the present study revealed grade 3 PSN with just a single dose. However, the incidence was similar to the result of a previous study, the severe PSN exacerbated the QOL of the patient during the treatment period (15). This should be taken into consideration when a investigator design the dosage or a longer schedule of nab-paclitaxel plus gemcitabine as a neoadjuvant therapy.

Patients who underwent surgery presented a stable course after receiving neoadjuvant therapy of nab-paclitaxel 
plus gemcitabine except for a patient who underwent DPCAR. This patient with DP-CAR had resection of both celiac axis and left inferior phrenic artery caused the necrotic ischemia especially in the fundic area of the stomach. It was most likely surgery-related SAE which would be overcome by the improvement of DP-CAR procedure $(18,26)$.

Another aim of this study was to evaluate the histopathologic efficacy of treatment as a neoadjuvant therapy for BRPC. Although the previous study reported high disease control rate, pancreatic tumors evaluable according to the RECIST (ver 1.1) were assessed as nontargeted lesions (15). In this study, we investigated the resection rate and the $\mathrm{R} 0$ resection rate by intention to treat, or the histopathologic response of the primary pancreatic tumor as the clinical outcomes of neoadjuvant therapy using nab-paclitaxel plus gemcitabine. This study demonstrated not only the safety and feasibility of nab-paclitaxel plus gemcitabine therapy for patients with BRPC, but the feasibility to obtain the data on resection rate, $\mathrm{R} 0$ resection rate, and the histopathologic response. However, this study demonstrated that resection and $\mathrm{R} 0$ rates were similar to previous studies (23), the relatively poor to moderate histopathologic response may depend on number of nabpaclitaxel plus gemcitabine administration.

In conclusion, the severe adverse drug reaction of neutropenia and PSN can affect the dose intensity or QOL for BRPC patients. In this study, we found that nabpaclitaxel plus gemcitabine therapy was safe and feasible without strict selection of patients with BRPC. We are planning a multicenter phase II study to investigate the efficacy of neoadjuvant nab-paclitaxel plus gemcitabine therapy on overall survival (UMIN000024154).

\section{Conflicts of Interest}

The Authors declare that they have no conflict of interest.

\section{Acknowledgements}

The Authors would like to thank the Department of Clinical Research Center, Wakayama Medical University, for proofreading and editing the manuscript. The study was carried out in accordance with the Declaration of Helsinki. The protocol was approved by the ethics committees of all participating institutions, and informed consent was obtained.

\section{References}

1 Konstantinidis IT, Warshaw AL, Allen JN, Blaszkowsky LS, Castillo CF, Deshpande V, Hong TS, Kwak EL, Lauwers GY, Ryan DP, Wargo JA, Lillemoe KD and Ferrone CR: Pancreatic ductal adenocarcinoma: is there a survival difference for R1 resections versus locally advanced unresectable tumors? What is a "true" R0 resection? Ann Surg 257: 731-736, 2013
2 Valle JW, Palmer D, Jackson R, Cox T, Neoptolemos JP, Ghaneh P, Rawcliffe CL, Bassi C, Stocken DD, Cunningham D, O'Reilly D, Goldstein D, Robinson BA, Karapetis C, Scarfe A, Lacaine F, Sand J, Izbicki JR, Mayerle J, Dervenis C, Oláh A, Butturini G, Lind PA, Middleton MR, Anthoney A, Sumpter K, Carter R and Büchler MW: Optimal duration and timing of adjuvant chemotherapy after definitive surgery for ductal adenocarcinoma of the pancreas: ongoing lessons from the ESPAC-3 study. J Clin Oncol 32: 504-512, 2014.

3 Katz MH, Pisters PW, Evans DB, Sun CC, Lee JE, Fleming JB, Vauthey JN, Abdalla EK, Crane CH, Wolff RA, Varadhachary GR and Hwang RF: Borderline resectable pancreatic cancer: the importance of this emerging stage of disease. J Am Coll Surg 206: 833-846, 2008.

4 Bockhorn M, Uzunoglu FG, Adham M, Imrie C, Milicevic M, Sandberg AA, Asbun HJ, Bassi C, Büchler M, Charnley RM, Conlon K, Cruz LF, Dervenis C, Fingerhutt A, Friess H, Gouma DJ, Hartwig W, Lillemoe KD, Montorsi M, Neoptolemos JP, Shrikhande SV, Takaori K, Traverso W, Vashist YK, Vollmer C, Yeo CJ and Izbicki JR; International Study Group of Pancreatic Surgery: Borderline resectable pancreatic cancer: a consensus statement by the International Study Group of Pancreatic Surgery (ISGPS). Surgery 155: 977-988, 2014.

5 Chatterjee D, Katz MH, Rashid A, Varadhachary GR, Wolff RA, Wang H, Lee JE, Pisters PW, Vauthey JN, Crane C, Gomez HF, Abbruzzese JL, Fleming JB and Wang H: Histologic grading of the extent of residual carcinoma following neoadjuvant chemoradiation in pancreatic ductal adenocarcinoma: a predictor for patient outcome. Cancer 118: 3182-3190, 2012.

6 Conroy T, Desseigne F, Ychou M, Bouché O, Guimbaud R, Bécouarn Y, Adenis A, Raoul JL, Gourgou-Bourgade S, de la Fouchardière C, Bennouna J, Bachet JB, Khemissa-Akouz F, Péré-Vergé D, Delbaldo C, Assenat E, Chauffert B, Michel P, Montoto-Grillot $\mathrm{C}$ and Ducreux M; Groupe Tumeurs Digestives of Unicancer; PRODIGE Intergroup: FOLFIRINOX versus gemcitabine for metastatic pancreatic cancer. N Engl J Med 364: 1817-1825, 2011.

7 Von Hoff DD, Ervin T, Arena FP, Chiorean EG, Infante J, Moore M, Seay T, Tjulandin SA, Ma WW, Saleh MN, Harris M, Reni M, Dowden S, Laheru D, Bahary N, Ramanathan RK, Tabernero J, Hidalgo M, Goldstein D, Van Cutsem E, Wei X, Iglesias J and Renschler MF: Increased survival in pancreatic cancer with nabpaclitaxel plus gemcitabine. N Engl J Med 369: 1691-1703, 2013.

8 Ferrone CR, Marchegiani G, Hong TS, Ryan DP, Deshpande V, McDonnell EI, Sabbatino F, Santos DD, Allen JN, Blaszkowsky LS, Clark JW, Faris JE, Goyal L, Kwak EL, Murphy JE, Ting DT, Wo JY, Zhu AX, Warshaw AL, Lillemoe KD and Fernández-del Castillo C: Radiological and surgical implications of neoadjuvant treatment with FOLFIRINOX for locally advanced and borderline resectable pancreatic cancer. Ann Surg 261: 12-17, 2015.

9 Hosein PJ, Macintyre J, Kawamura C, Maldonado JC, Ernani V, Loaiza-Bonilla A, Narayanan G, Ribeiro A, Portelance L, Merchan JR, Levi JU and Rocha-Lima CM: A retrospective study of neoadjuvant FOLFIRINOX in unresectable or borderlineresectable locally advanced pancreatic adenocarcinoma. BMC Cancer 12: 199, 2012.

10 Christians KK, Tsai S, Mahmoud A, Ritch P, Thomas JP, Wiebe L, Kelly T, Erickson B, Wang H, Evans DB and George B: Neoadjuvant FOLFIRINOX for borderline resectable pancreas cancer: a new treatment paradigm? Oncologist 19: 266-274, 2014. 
11 Mahaseth H, Brutcher E, Kauh J, Hawk N, Kim S, Chen Z, Kooby DA, Maithel SK, Landry J and El-Rayes BF: Modified FOLFIRINOX regimen with improved safety and maintained efficacy in pancreatic adenocarcinoma. Pancreas 42: 1311-1315, 2013.

12 Blazer M, Wu C, Goldberg RM, Phillips G, Schmidt C, Muscarella P, Wuthrick E, Williams TM, Reardon J, Ellison EC, Bloomston $\mathrm{M}$ and Bekaii-Saab T: Neoadjuvant modified (m) FOLFIRINOX for locally advanced unresectable (LAPC) and borderline resectable (BRPC) adenocarcinoma of the pancreas. Ann Surg Oncol 22: 1153-1159, 2015.

13 Okada K, Kawai M, Hirono S, Satoi S, Yanagimoto H, Ioka T, Miyazawa M, Shimizu A, Kitahata Y and Yamaue H: Impact of treatment duration of neoadjuvant FIRINOX in patients with borderline resectable pancreatic cancer: a pilot trial. Cancer Chemother Pharmacol 78: 719-726, 2016.

14 Hirono S, Kawai M, Okada K, Miyazawa M, Shimizu A, Kitahata Y, Ueno $M$ and Yamaue H: Treatment strategy for borderline resectable pancreatic cancer with radiographic artery involvement. Pancreas 45: 1438-1446, 2016.

15 Ueno $\mathrm{H}$, Ikeda $\mathrm{M}$, Ueno M, Mizuno N, Ioka T, Omuro Y, Nakajima TE and Furuse J: Phase I/II study of nab-paclitaxel plus gemcitabine for chemotherapy-naive Japanese patients with metastatic pancreatic cancer. Cancer Chemother Pharmacol 77: 595-603, 2016.

16 National Comprehensive Cancer Network: Clinical Practice Guidelines in Oncology. Pancreatic adenocarcinoma. Version 1. 2014.

17 Hirano S, Kondo S, Hara T, Ambo Y, Tanaka E, Shichinohe T, Suzuki O and Hazama K: Distal pancreatectomy with en bloc celiac axis resection for locally advanced pancreatic body cancer: long-term results. Ann Surg 246: 46-51, 2007.

18 Okada K, Kawai M, Tani M, Hirono S, Miyazawa M, Shimizu A, Kitahata Y and Yamaue H: Surgical strategy for patients with pancreatic body/tail carcinoma: who should undergo distal pancreatectomy with en-bloc celiac axis resection? Surgery 153 : 365-372, 2013.

19 Huang HQ, Brady MF, Cella D and Fleming G: Validation and reduction of FACT/GOG-Ntx subscale for platinum/paclitaxelinduced neurologic symptoms: a gynecologic oncology group study. Int J Gynecol Cancer 17: 387-393, 2007.
20 Evans DB, Rich TA, Byrd DR, Cleary KR, Connelly JH, Levin B, Charnsangavej C, Fenoglio CJ and Ames FC: Preoperative chemoradiation and pancreaticoduodenectomy for adenocarcinoma of the pancreas. Arch Surg 127: 1335-1339, 1992.

21 Common Terminology Criteria for Adverse Events (CTCAE) Version 4.0 Published: May 28, 2009 (v4.03: June 14, 2010) U.S. Department of Health and Human Services National Institutes of Health, National Cancer Institute.

22 Dindo D, Demartines $\mathrm{N}$ and Clavien PA: Classification of surgical complications: a new proposal with evaluation in a cohort of 6336 patients and results of a survey. Ann Surg 240: 205-213, 2004.

23 Gillen S, Schuster T, Meyer Zum Büschenfelde C, Friess H and Kleeff J: Preoperative/neoadjuvant therapy in pancreatic cancer: a systematic review and meta-analysis of response and resection percentages. PLoS Med 7: e1000267, 2010.

24 Okusaka T, Ikeda M, Fukutomi A, Ioka T, Furuse J, Ohkawa S, Isayama $\mathrm{H}$ and Boku N: Phase II study of FOLFIRINOX for chemotherapy-naïve Japanese patients with metastatic pancreatic cancer. Cancer Sci 105: 1321-1326, 2014.

25 Scheithauer W, Ramanathan RK, Moore M, Macarulla T, Goldstein D, Hammel P, Kunzmann V, Liu H, McGovern D, Romano A and Von Hoff DD: Dose modification and efficacy of nab-paclitaxel plus gemcitabine $v s$. gemcitabine for patients with metastatic pancreatic cancer: phase III MPACT trial. J Gastrointest Oncol 7: 469-478, 2016.

26 Okada K, Kawai M, Tani M, Hirono S, Miyazawa M, Shimizu A, Kitahata Y and Yamaue H: Preservation of the left gastric artery on the basis of anatomical features in patients undergoing distal pancreatectomy with celiac axis en-bloc resection (DPCAR). World J Surg 38: 2980-2985, 2014. 